\title{
Norois
}

Environnement, aménagement, société

\section{Des indices pour hiérarchiser la sensibilité du littoral aux pollutions marines par les hydrocarbures : l'exemple normand}

Indexes to assess the coastal zone sensitivity to marine pollution: the case of Normandy

Iwan Le Berre, Mohamed Rassim Hariz, Laurence David et Loïc Nogues

\section{OpenEdition}

Journals

\section{Édition électronique}

URL : https://journals.openedition.org/norois/3607

DOI : $10.4000 /$ norois.3607

ISSN : $1760-8546$

\section{Éditeur}

Presses universitaires de Rennes

\section{Édition imprimée}

Date de publication : 30 juin 2011

Pagination : 109-129

ISBN : 978-2-7535-1479-9

ISSN : 0029-182X

\section{Référence électronique}

Iwan Le Berre, Mohamed Rassim Hariz, Laurence David et Loïc Nogues, « Des indices pour hiérarchiser la sensibilité du littoral aux pollutions marines par les hydrocarbures : l'exemple normand », Norois [En ligne], 219 | 2011, mis en ligne le 30 septembre 2013, consulté le 13 janvier 2022. URL : http://journals.openedition.org/norois/3607 ; DOI : https://doi.org/10.4000/norois.3607

Ce document a été généré automatiquement le 13 janvier 2022.

(c) Tous droits réservés 


\title{
Des indices pour hiérarchiser la sensibilité du littoral aux pollutions marines par les hydrocarbures: l'exemple normand
}

\author{
Indexes to assess the coastal zone sensitivity to marine pollution: the case of \\ Normandy
}

Iwan Le Berre, Mohamed Rassim Hariz, Laurence David et Loïc Nogues

1 L'organisation française de lutte contre les pollutions accidentelles marines constitue un volet spécifique du plan ORSEC départemental ${ }^{1,2}$. Ces plans POLMAR théoriquement révisés tous les cinq ans (CEDRE, 2003), doivent notamment fournir « un inventaire précis et hiérarchisé des zones à protéger en priorité ». Etabli sous la forme d'un atlas de sensibilité des littoraux, annexe obligatoire du Plan POLMAR, cet inventaire est un document synthétique d'aide à la décision dans le contexte préparatoire et opérationnel de la lutte antipollution.

2 La nature et la quantité de polluant, les conditions météorologiques et océaniques, ainsi que la morphologie du littoral déterminent les conditions d'échouage et les lieux préférentiels de dépôt du pétrole à la côte. Ils définissent aussi largement les conditions et la faisabilité de l'intervention. De plus, la nature du littoral, son patrimoine écologique et les activités et aménagements qui s'y sont implantés déterminent la sensibilité et la vulnérabilité locale. C'est donc un ensemble complexe de paramètres qui influence le choix, des priorités d'intervention, des techniques et des matériels de lutte à mettre en œuvre lors d'une intervention.

3 On distingue généralement les paramètres conjoncturels - nature et contexte de la pollution - des paramètres structurels : caractéristiques environnementales et socioéconomiques du littoral affecté (Clark, 2002 ; Lacaze, 1980). Les premiers font le plus souvent l'objet d'une expertise en situation de crise, notamment sur le terrain, tandis que les seconds se prêtent davantage à un inventaire hiérarchisé utile pour alimenter 
les atlas de sensibilité conçus en amont, dans une perspective de prévention et de planification.

4 Cependant, si la plupart des données utiles pour la préparation d'une intervention existent, elles ne sont pas toujours disponibles et directement mobilisables en situation de crise. De plus, leur caractère hétérogène, lié à la grande diversité de leurs sources et de leurs formats, ne facilite pas leur consultation ni leur analyse. L'objectif des atlas de sensibilité est donc de rassembler sous une forme cohérente les données pertinentes disponibles.

L'analyse des atlas existants dans les départements littoraux français montre que l'hétérogénéité reste de mise: les contextes, les enjeux, les moyens alloués, et les maîtres d'œuvre diffèrent d'un département à l'autre (Le Berre et al., 2008). Les méthodes employées pour la hiérarchisation des enjeux présentent également une variabilité importante, qui peut pénaliser la préparation d'une intervention en limite de départements. En tirant partie de l'expérience acquise pour la mise à jour des trois atlas normands, cet article propose des éléments de réflexion pour l'harmonisation des atlas de sensibilité, particulièrement importante en cas de pollution dépassant le cadre départemental, voire régional, comme dans le cas d'estuaires (Seine, Gironde) ou de baies (Mont-Saint-Michel, baie des Veys) artificiellement découpés par des limites administratives.

\section{Contexte et méthode}

\section{La Mimel}

6 Notre réflexion s'inscrit dans le cadre de l'étude Information géographique et Gestion intégrée de la zone côtière réalisée entre les mois d'octobre 2008 et 2010 par GÉOMER pour le compte de la Mission Interservices de la Mer et du Littoral (Le Berre et al., 2010). La MIMEL est une création du Comité Interministériel d'Aménagement et de Développement $\mathrm{du}$ Territoire (CIADT) du 14 septembre 2004. Administration expérimentale, elle avait ${ }^{3}$ pour vocation de renforcer la coordination et la transversalité des services et établissements publics de l'État, dans leur appréhension des questions littorales et maritimes, dans un souci de cohérence des politiques publiques de l'État en mer en concertation avec les différents usagers de l'espace marin.

7 L'étude confiée à GÉOMER visait à développer, sur les régions de Basse et de HauteNormandie, un pôle géomatique Mer et Littoral doté d'une triple vocation :

- structurer et mutualiser l'information géographique pour en améliorer la circulation entre les différents services, notamment par la standardisation des protocoles de collecte et de traitement des données;

- compléter et valoriser l'information géographique existante sur la mer et le littoral ;

- proposer une analyse prospective en développant des méthodes de travail adaptées à l'évolution rapide des enjeux du littoral.

8 Une application de ce pôle a consisté en l'exploitation de l'information géographique collectée auprès des services de l'État et des autres partenaires de la MIMEL pour produire et mettre à jour les atlas POLMAR-TERRE des départements côtiers des deux régions normandes : Manche, Calvados et Seine-Maritime (fig. 1). 


\section{Méthodologie}

La première étape de notre démarche a consisté à rassembler les sources documentaires utiles pour alimenter notre réflexion. Cette collecte s'est appuyée sur une synthèse de la littérature scientifique réalisée en partenariat avec le CEDRE (Le Berre et al., 2005), complétée et actualisée dans le cadre de cette étude. Nous avons également recherché et collecté auprès des services de l'État (DDE/DDTM, DIREN/DREAL) les atlas POLMAR disponibles en France métropolitaine et dans les DOM-TOM (13 atlas ont été obtenus sur les 21 identifiés). La structuration de ces atlas, les méthodes de représentation et de hiérarchisation des enjeux du littoral et de leur sensibilité à la pollution marine, ainsi que la nature et la source des données exploitées pour ce faire ont été analysées (Le Berre et al., 2008).

Cette analyse met en évidence la forte hétérogénéité des atlas produits qui s'exprime à la fois dans le fond (quantité et qualité de l'information géographique utilisée), dans les méthodologies mises en œuvre (choix des indices, utilisation ou pas des systèmes d'information géographique) et dans la forme (représentation cartographique, échelles et nombre de cartes). Elle trouve son explication dans plusieurs causes. En premier lieu, ces documents ont été produits à des époques différentes et répondent de ce fait à des cahiers des charges officiels différents ${ }^{4}$. En second lieu, le guide de révision des Plans POLMAR-TERRE (CEDRE, 2003) indique les grandes lignes du contenu et de la forme des atlas de sensibilité, mais ne propose pas de préconisations méthodologiques détaillées, ni de charte cartographique pour leur préparation. Enfin, la maîtrise d'ouvrage et les investissements consacrés à la révision des atlas sont très variables d'un département à l'autre. On a ainsi distingué trois types d'atlas, du plus minimaliste au plus élaboré (Le Berre et al., 2008).

11 Cette base nous a ensuite permis d'engager une consultation des services de l'État intervenant en cas de déclenchement du plan POLMAR-TERRE, et d'experts de la lutte antipollution de plusieurs organismes (CEDRE, CETMEF, UBO, etc.) afin de définir l'organisation et le contenu à donner aux atlas POLMAR de Normandie. Les données nécessaires pour produire les atlas ont ensuite été collectées au sein des services de l'État et auprès des partenaires de la MIMEL. Certaines ont été complétées sur le terrain. Toutes ces données ont été intégrées au sein du SIG développé pour le compte de la MIMEL, puis ont été analysées et cartographiées pour alimenter les atlas. 
Figure 1 : Présentation de la zone d'étude/Location map

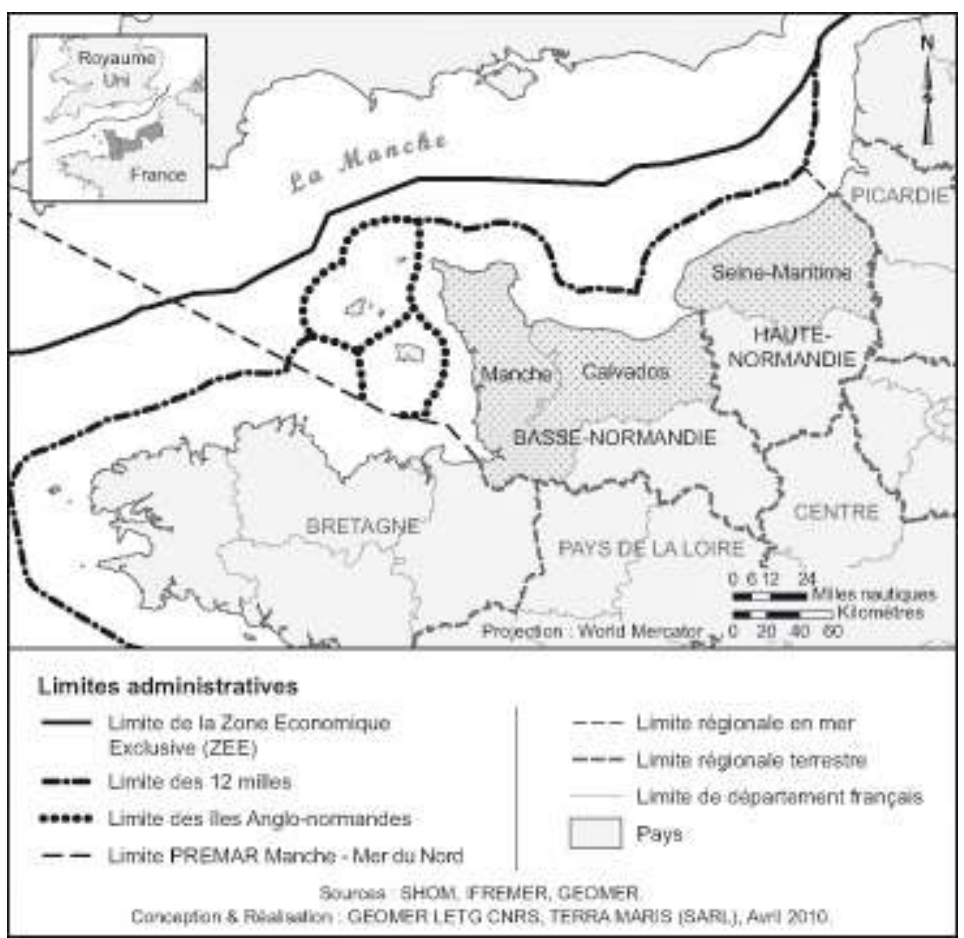

C'est donc à la suite d'expérimentations successives, soumises à expertise, amendées puis validées, que les trois atlas normands ont été mis à jour sous une forme harmonisée tant par leur structure, que par les données employées et les modes de représentation et de hiérarchisation de la sensibilité du littoral aux pollutions marines. C'est le fruit de cette réflexion qui est livré dans cet article.

\section{Des indices variés pour exprimer la sensibilité}

Les atlas de sensibilité doivent présenter une synthèse cartographique des enjeux du littoral (Bastien-Ventura, 2005). Les méthodes d'évaluation et de représentation de ces enjeux varient selon les atlas. Elles s'appuient en particulier sur l'utilisation d'indices définis et calculés par des méthodes diverses: qualitatives, quantitatives, non spatialisées ou spatialisées (Fattal, 2008). Les indices peuvent être thématiques - on distingue alors communément des indices géomorphologiques (Gundlach et Hayes, 1978 ; Michel et Dahlin, 1997), écologiques ${ }^{5}$ et socio-économiques (Levratto et Clémenceau, 2005) - ils peuvent combiner les thématiques géomorphologique et écologique (Kerambrun et al., 1996), voire les trois thématiques (Lagabrielle, 2001; Fattal, 2008 ; Castanedo et al., 2009). La présentation réalisée dans cette partie concerne essentiellement les approches développées sur les littoraux français pour l'élaboration des atlas POLMAR-TERRE. Pour une revue plus complète de la littérature internationale consulter l'ouvrage de P. Fattal (2008).

\section{Un indice morphosédimentaire communément employé}

14 L'analyse des atlas réalisés montre que, sur les trois volets de sensibilité communément développés, seul l'indice de sensibilité géomorphologique fait l'objet d'un large 
consensus. L'ESI (Environmental Sensitivity Index) défini dans les années 1970 par E. R. Gundlach et M. O. Hayes (1978) sur la base de critères morphosédimentaires a fait l'objet de nombreuses adaptations locales en France, comme dans de nombreuses parties du monde, en fonction des types de milieux (estuariens, lacustres, etc.) et des latitudes : tropicales, tempérées, polaires (Michel et Dahlin, 1997; NOAA, 2002 ; OMI/ IPECA, 2007 ; Le Berre et al., 2005).

L'ESI tient compte de la nature morphologique et sédimentaire (granulométrie) de l'estran et du trait de côte (niveau des plus hautes mers de vives-eaux, c'est-à-dire susceptible d'être atteint par une pollution marine), ainsi que de leur exposition aux agents hydrodynamiques (houles, courants). Ces deux facteurs déterminent la capacité de piégeage des sédiments, matérialisée par la rémanence des hydrocarbures, et la capacité d'auto-nettoyage du site. Ainsi, les sédiments grossiers favorisent la percolation des hydrocarbures, leur stockage et leur relargage périodique, prolongeant dans le temps l'impact de la pollution. Par ailleurs, c'est principalement le mode d'exposition de la côte (battu ou abrité) qui détermine la capacité d'auto-nettoyage des milieux, donc la persistance de la pollution.

Il convient cependant de remarquer que l'ESI a été établi sur la base de l'analyse des impacts de cinq accidents intervenus au début des années 1970 qui mettaient tous en jeu des hydrocarbures légers non représentatifs de toute la gamme de polluants susceptibles d'être déversés en mer (Little et al., 2003). Or, le comportement, le piégeage et la rémanence des hydrocarbures sur les côtes sont non seulement fonction des caractéristiques hydromorphosédimentaires du milieu, mais aussi de la nature du polluant et en particulier de sa viscosité et de son adhérence au substrat (Dicks et al., 2002). Pour ne prendre que l'exemple des deux dernières marées noires européennes d'importance majeure, celles de l'Erika en 1999 et du Prestige en 2003, dont les déversements étaient composés d'hydrocarbures lourds ou résiduels très visqueux, les rémanences indiquées par l'ESI ne conviennent pas (Fichaut et Fattal, 2000). Ainsi, les falaises, fussent-elles très exposées (indice 1 sur l'ESI), ont fait l'objet d'un nettoyage long, fastidieux, coûteux sans lequel la pollution aurait eu une rémanence de plusieurs années sans aucun doute supérieure à celle des plages de sable fin à grossiers (ESI 3, 4).

17 A part dans les publications qui viennent d'être citées, ces limites sont dans les faits rarement soulignées et ne remettent pas en cause l'ESI qui reste la référence la plus communément adoptée. Cela s'explique par la simplicité de compréhension de cet indice et de sa logique a priori irréprochable, mais aussi car en général lors d'une intervention les experts en nettoyage travaillent et adaptent leurs techniques en fonction du contexte toujours particulier de la pollution à traiter.

18 Le tableau 1 montre un exemple d'adaptation locale de cet indice pour l'atlas de sensibilité du littoral de la Manche (50).

19 Tableau 1 : Indice de sensibilité morphosédimentaire adapté au littoral de la Manche/ The Manche coastal zone Environmental Sensitivity Index

\begin{tabular}{|l|l|l|l|l|l|}
\hline $\begin{array}{l}\text { n/ } \\
\text { A }\end{array}$ & Énergie & Typologie agregee & Typologie détaillée & $\begin{array}{l}\text { Durée de la } \\
\text { pollution }\end{array}$ & esi \\
\hline $\mathrm{N}$ & $\begin{array}{l}\text { côtes exposées } \\
\text { (haute énergie) }\end{array}$ & côtes à falaises & falaises rocheuses & $\begin{array}{l}\text { quelques } \\
\text { semaines }\end{array}$ & 1 \\
\cline { 2 - 5 } & &
\end{tabular}




\begin{tabular}{|c|c|c|c|c|c|}
\hline $\mathrm{N}$ & & & falaises meubles & $\begin{array}{l}\text { quelques } \\
\text { semaines }\end{array}$ & 1 \\
\hline \multirow[t]{2}{*}{$\mathrm{N}$} & & \multirow[t]{2}{*}{ platiers rocheux } & $\begin{array}{l}\text { platiers rocheux, plateformes } \\
\text { d'érosion }\end{array}$ & $\begin{array}{l}\text { quelques } \\
\text { mois }\end{array}$ & 2 \\
\hline & & & champ de blocs & 2 à 3 ans & 4 \\
\hline $\mathrm{N}$ & & \multirow{3}{*}{ estrans sableux } & sables fins à moyens, dunes & 1 à 2 ans & 3 \\
\hline $\mathrm{N}$ & & & sables grossiers & 2 à 3 ans & 4 \\
\hline $\mathrm{N}$ & & & sables et graviers & 3 à 5 ans & 4 \\
\hline $\mathrm{N}$ & & \multirow{2}{*}{$\begin{array}{l}\text { estrans sédimentaires } \\
\text { hétérogènes }\end{array}$} & $\begin{array}{l}\text { sables fins à grossiers, } \\
\text { éventails deltaïques }\end{array}$ & 3 à 5 ans & 5 \\
\hline $\mathrm{N}$ & & & $\begin{array}{l}\text { sédiments hétérogènes, sables } \\
\text { moyens, graviers }\end{array}$ & 3 à 5 ans & 5 \\
\hline $\mathrm{N}$ & & galets et graviers & galets et graviers & 3 à 5 ans & 6 \\
\hline A & & \multirow{3}{*}{$\begin{array}{l}\text { côtes artificielles } \\
\text { imperméables }\end{array}$} & $\begin{array}{l}\text { murs de défense, perrés } \\
\text { maçonnés }\end{array}$ & & 1 \\
\hline A & & & $\begin{array}{l}\text { portes à flot, écluses et } \\
\text { barrages, ponts }\end{array}$ & & 1 \\
\hline A & & & épis en dur & & 3 \\
\hline A & & \multirow{4}{*}{$\begin{array}{l}\text { côtes artificielles } \\
\text { perméables }\end{array}$} & cordons d'enrochements & & 6 \\
\hline A & & & épis en enrochements & & 6 \\
\hline A & & & $\begin{array}{l}\text { alternance murs/cordons } \\
\text { d'enrochements }\end{array}$ & & 6 \\
\hline A & & & caisson de jarlan & & 6 \\
\hline $\mathrm{N}$ & \multirow{13}{*}{$\begin{array}{l}\text { côtes abritées } \\
\text { (faible énergie) }\end{array}$} & côte rocheuse & côte rocheuse & 3 à 5 ans & 6 \\
\hline $\mathrm{N}$ & & \multirow{2}{*}{ estrans sableux } & sables fins à moyens, dunes & $>5$ ans & 7 \\
\hline $\mathrm{N}$ & & & sables grossiers à graviers & $>5$ ans & 7 \\
\hline $\mathrm{N}$ & & galets et graviers & galets et graviers & $>5$ ans & 8 \\
\hline $\mathrm{N}$ & & vasières & $\begin{array}{l}\text { vasières, sédiments sablo- } \\
\text { vaseux }\end{array}$ & $>10$ ans & 9 \\
\hline $\mathrm{N}$ & & marais maritimes & marais maritimes & $>10$ ans & 10 \\
\hline $\mathrm{N}$ & & récifs d'hermelles & banc d'hermelles & $>10$ ans & 10 \\
\hline $\mathrm{N}$ & & berges fluviales & berges fluviales & $>10$ ans & 10 \\
\hline A & & \multirow{4}{*}{$\begin{array}{l}\text { côtes artificielles } \\
\text { imperméables }\end{array}$} & épis en dur & & 6 \\
\hline A & & & murs, quais, perrés maçonnés & & 6 \\
\hline A & & & digues, digues végétalisées & & 6 \\
\hline A & & & $\begin{array}{l}\text { portes à flot, écluses et } \\
\text { barrages, ponts }\end{array}$ & & 6 \\
\hline A & & $\begin{array}{l}\text { côtes artificielles } \\
\text { perméables }\end{array}$ & $\begin{array}{l}\text { cordons d'enrochements, épis } \\
\text { en enrochements }\end{array}$ & & 8 \\
\hline
\end{tabular}




\begin{tabular}{|l|l|l|l|l|l|}
\hline A & \begin{tabular}{l|l|l|l|} 
alternance murs et \\
enrochements
\end{tabular} & 8 \\
\hline A & caisson de jarlan & & 8 \\
\hline
\end{tabular}

N/A : littoral Artificiel ou Naturel ; ESI : Environmental sensitivity index, indice de sensibilité morphosédimentaire croissant de 1 (faible sensibilité) à 10 (forte sensibilité)

(d'après S. Berné et al., 1982 ; E. R. Gundlach et M.O. Hayes, 1978)

L'indice peut être représenté sous forme linéaire, les différentes catégories étant reportées sur un trait de côte. C'est le cas de la plupart des atlas ESI (voir par exemple celui de l'Afrique centrale et de l'ouest) ${ }^{6}$ qui sont présentés à de petites échelles (inférieures à $1: 100000$ ). À plus grande échelle (du 1:50 000 au 1:10 000) les entités morphosédimentaires sont représentées sous forme de surfaces dont la délimitation requiert des données beaucoup plus détaillées. Pour les atlas normands les deux méthodes ont été retenues : représentation linéaire pour les cartes départementales, surfacique pour les cartes détaillées (1:50 000).

\section{Plusieurs méthodes d'évaluation de la sensibilité écologique}

Plusieurs indices sont employés pour décrire la sensibilité écologique. Leur mise en œuvre dépend de l'échelle de représentation (départementale, locale), mais aussi du type et du niveau de précision des données de référence disponibles en relation avec cette thématique.

- Niveau de protection et intérêt patrimonial : cet indice repose sur la somme des dispositifs réglementaires et contractuels, et des inventaires appliqués aux sites naturels en fonction de leur importance paysagère, de leur représentativité, de leur rareté et de leur sensibilité. Relativement aisé à produire par des méthodes d'analyse spatiale classiques dans un SIG, à partir des données sur les zonages environnementaux désormais téléchargeables sur les sites web des DREAL, il a été appliqué dans le Finistère $(2005)^{7}$, dans le Pas-de-Calais $(2004)^{8}$ et pour les atlas de Haute et de BasseNormandie $(2009,2010)$. La note finale attribuée à un territoire dépend du nombre de zonages qui s'y superposent. Elle permet ainsi d'évaluer la valeur patrimoniale attribuée à ce territoire. Une pondération peut être proposée en fonction d'une cotation définie à dires d'experts, comme dans l'exemple de la Basse-Normandie présenté dans le tableau 2.

Tableau 2 : Cotation des zonages environnementaux définie par la DREAL de Basse-Normandie/ Quotation of protected areas as defined by the DREAL of Basse-Normandie

\begin{tabular}{|l|l|}
\hline Nature de protection & Cotation Diren \\
\hline Site d'Intérêt communautaire (SIC), Directive Habitats & 4 \\
\hline Zone de Protection Spéciale (ZPS), Directive Oiseaux & 4 \\
\hline Proposition de Sites d'Intérêt Communautaire (pSIC), Directive Oiseaux & 4 \\
\hline Site classé & 4 \\
\hline Réserve Naturelle Nationale & 4 \\
\hline Arrêté Préfectoral de Protection de Biotope & 3 \\
\hline
\end{tabular}




\begin{tabular}{|l|l|}
\hline Réserve Naturelle Régionale & 3 \\
\hline Site inscrit & 3 \\
\hline Espaces naturels sensibles (terrains du Conservatoire, des collectivités) & 2 \\
\hline ZNIEFF de type 1 & 2 \\
\hline Site Ramsar & 1 \\
\hline ZNIEFF de type 2 & 1 \\
\hline Zones humides & 1 \\
\hline
\end{tabular}

Figure 2 : Exemple de carte de la valeur patrimoniale (superposition des protections dans le département de la Manche)/Heritage assessment based on the sum of the protected areas of the department of Manche

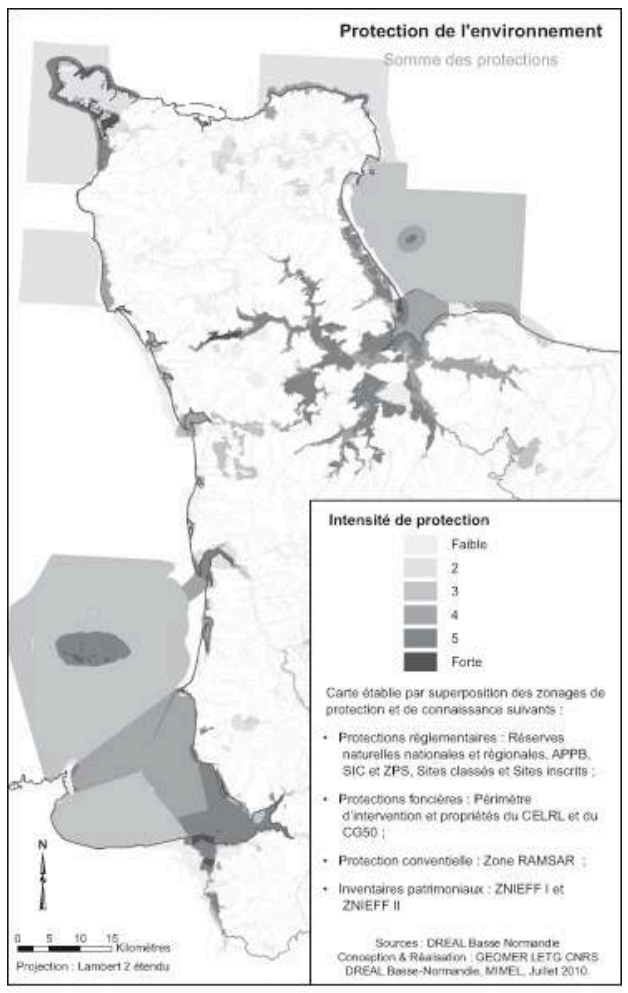

- Habitats naturels côtiers : Suite à l'accident de l'Erika en 1999, et fort de son expérience dans le domaine de la cartographie des habitats Natura 2000 (Hardegen et al., 2001), le Conservatoire Botanique National de Brest (CBNB) a entrepris la réalisation d'un état de référence des habitats littoraux'. À partir de celui-ci est dérivé un indice qui s'appuie sur la valeur patrimoniale des espèces végétales, l'impact direct des polluants sur ces espèces et l'impact des opérations de dépollution sur les habitats. Il est constitué de quatre classes indiquant la sensibilité des habitats à une pollution marine mais également aux opérations de nettoyage. Cet indice a déjà été utilisé dans les atlas POLMAR du Pas-de-Calais en 2004, de Loire-Atlantique en 2004, du Finistère en 2005, de Haute et de Basse-Normandie en 2010. Son application repose sur une description détaillée (de type phytosociologique) des habitats naturels côtiers et de leur état de conservation, non encore disponible sur l'ensemble du littoral français - seules 
la Bretagne et la Normandie ayant été cartographiées pour l'heure - et d'une expertise permettant d'établir leur cotation.

- La faune peut également donner lieu à une représentation particulière et en premier lieu l'avifaune considérée comme particulièrement sensible aux pollutions marines. Dans certains cas, les sites colonisés par les espèces les plus s ensibles sont simplement localisés et représentés par des symboles ponctuels (pictogrammes). Cette méthode est employée dans les atlas de type $\mathrm{ESI}^{10}$, ainsi que sur les cartes détaillées des atlas du Nord et du Pas-de-Calais en 2004. Les cartographies sont parfois complétées par des listes d'espèces. Cette représentation ne permet cependant pas de hiérarchiser les enjeux. Dans d'autres cas, les suivis ornithologiques réguliers menés par les scientifiques ou par des associations environnementalistes sont exploités pour produire une cartographie synthétique hiérarchisant les sites en fonction de leur intérêt patrimonial et/ou fonctionnel. Ce type de travail a été réalisé notamment dans le Finistère en 2005, en collaboration avec Bretagne-Vivante. En Normandie, le СBNB a travaillé entre 2006 et 2008 avec le Conservatoire du littoral dans le cadre du programme ATRACTIV ${ }^{11}$ qui associait également plusieurs associations dont le Groupe ornithologique normand (GON). Cette collaboration visait la mise en place d'un état de référence naturaliste incluant l'avifaune et les mammifères marins ainsi que la production d'une cartographie fine et d'une hiérarchisation de la vulnérabilité des habitats naturels côtiers du littoral normand. Les paramètres employés sont présentés à titre d'exemple dans le tableau 3.

Tableau 3 : Critères fonctionnels, patrimoniaux et quantitatifs pour évaluer la sensibilité de l'avifaune aux pollutions marines/Criterions for the assessment of marine birds sensitivity to marine pollution

\begin{tabular}{|l|l|l|}
\hline Paramètre du site & Modalité & $\begin{array}{l}\text { Variabilité } \\
\text { temporelle }\end{array}$ \\
\hline Espèces présentes & $\begin{array}{l}\text { Liste des espèces présentes/liste d'espèces } \\
\text { sélectionnées/espèces ou groupes d'espèces } \\
\text { indicatrices }\end{array}$ & $\begin{array}{l}\text { Saisonnière/ } \\
\text { annuelle }\end{array}$ \\
\hline $\begin{array}{l}\text { Statut réglementaire des } \\
\text { espèces présentes }\end{array}$ & $\begin{array}{l}\text { Inscription dans des listes ou annexes de textes de } \\
\text { loi (internationaux, européens, français) }\end{array}$ & $\begin{array}{l}\text { Susceptible } \\
\text { d'évoluer }\end{array}$ \\
\hline Statut de conservation & $\begin{array}{l}\text { Inscription sur les Listes Rouge et Orange } \\
\text { (mondiale, nationale, régionale) }\end{array}$ & $\begin{array}{l}\text { Susceptible } \\
\text { d'évoluer }\end{array}$ \\
\hline Effectifs par espèce & Nombre d'individus ou de couples & $\begin{array}{l}\text { Saisonnière/ } \\
\text { annuelle }\end{array}$ \\
\hline $\begin{array}{l}\text { Contribution relative par } \\
\text { espèce }\end{array}$ & $\begin{array}{l}\text { Proportion par rapport à un effectif mondial, } \\
\text { européen, régional }\end{array}$ & $\begin{array}{l}\text { Saisonnière/ } \\
\text { annuelle }\end{array}$ \\
\hline Fréquence & Fréquence d'occupation du site par l'espèce & $\begin{array}{l}\text { Saisonnière/ } \\
\text { annuelle }\end{array}$ \\
\hline Fonction & Reproduction, alimentation, repos, etc. & $\begin{array}{l}\text { Saisonnière/ } \\
\text { annuelle }\end{array}$ \\
\hline
\end{tabular}

(Binard et al., 2008) 
Ainsi la sensibilité écologique peut-elle être définie par plusieurs indices dont l'échelle d'application diffère en fonction du niveau de détail souhaité. La superposition des zonages de protection environnementale permet d'apprécier la valeur patrimoniale à l'échelle départementale. Elle a une valeur essentiellement stratégique. Les indices de sensibilité des habitats et de l'avifaune s'appliquent à une échelle locale. Ils ont par conséquent une vocation plus opérationnelle notamment en vue de la mise en œuvre des chantiers de dépollution.

\section{Définir la sensibilité socio-économique...}

La sensibilité des activités économiques est essentiellement due à la destruction de matériels, à la contamination des productions et à l'impossibilité de pratiquer l'activité. Aucun indice ne fait actuellement l'objet d'un consensus pour exprimer la sensibilité socio-économique des littoraux, chaque atlas adoptant sa propre méthodologie en fonction des enjeux en présence, des données disponibles et de son maitre d'œuvre. On peut distinguer plusieurs catégories de méthodes :

27 - Les principales activités et certains aménagements sont simplement listés et localisés sur le littoral considéré. C'est par exemple le cas dans les premières versions des atlas POLMAR de la Manche en 1996 et de la Seine-Maritime en 1999. L'avantage de cette méthode réside dans sa légèreté de mise en œuvre qui repose sur la mobilisation de données relativement aisées à obtenir auprès des DREAL (liste des industries classées à risque) et des DDTM (Autorisation d'occupation temporaire du Domaine public maritime pour les concessions de cultures et d'élevages marins, les prises d'eau, etc.). En revanche, elle ne propose qu'un inventaire non hiérarchisé.

28 - L'inventaire peut prendre en compte toutes les activités du littoral pouvant être affectées par une pollution marine: industrielles, portuaires, aquacoles, mais également touristiques et commerciales. Il nécessite de solliciter des acteurs variés : conseils généraux et comités départementaux de tourisme, sections conchylicoles et comités des pêches locaux ou régionaux, etc. L'inventaire peut ne s'intéresser qu'aux activités professionnelles ou, comme dans l'atlas de la Corse (Levratto et Clémenceau, 2005), intégrer également les usages non marchands considérant alors leur valeur de bien collectif. Dans ce cas, des enquêtes complémentaires sur le terrain sont nécessaires. En l'absence d'un dispositif pérenne d'observation, elles peuvent compliquer les mises à jour ultérieures de l'atlas.

29 - Les activités peuvent être cartographiées sous forme de symboles ponctuels, à l'exemple des atlas de la Manche en 1996, de la Seine-Maritime en 1999, du Calvados en 2000, du Finistère en 2005 ou du Morbihan en 2005. Cela permet de les localiser précisément, mais peut rapidement se traduire par une saturation d'information quand les activités sont nombreuses. Une autre possibilité réside dans leur représentation surfacique: emprise des établissements inventoriés ou, plus couramment, limites communales. L'utilisation de ces dernières offre l'avantage de permettre l'exploitation directe de la plupart des statistiques socio-économiques disponibles auprès des services de l'État, des collectivités territoriales et de l'INSEE notamment. En revanche, cette méthode se traduit par une agrégation des données à l'échelle communale. Cette méthode a cependant été retenue pour les atlas du Nord et du Pas-de-Calais en 2004 et de la Corse en 2005 car elle permet une hiérarchisation du littoral à l'échelle d'un département. Les deux approches peuvent du reste être employées de manière 
complémentaire au sein d'un même atlas : intégration par commune pour les cartes départementales, localisation des activités par leur emprise spatiale (zones conchylicoles par exemple) ou par un symbole ponctuel (prises d'eau) à l'échelle locale.

- Le traitement des données peut aller de la simple somme des activités présentes à l'échelle communale (seconde version de l'atlas de la Manche en 2006), jusqu'à l'utilisation de processus de calcul plus complexes, faisant appel à un système de pondération destiné à hiérarchiser entre elles les activités considérées (Levratto et Clémenceau, 2005). L'importance des activités s'apprécie alors à partir de données socio-économiques sur l'emploi, la fréquentation, le chiffre d'affaires, etc. Selon les activités concernées, selon les sources à solliciter (administrations, entreprises), et en raison de leur caractère souvent stratégique ou confidentiel, ces données peuvent être difficiles à rassembler, mais également à combiner (Le Sage, 2000). Ce type d'approche est surtout employé pour l'évaluation des dommages causés par une marée noire (Bonnieux et Rainelli, 2002).

31 - Lors d'une pollution marine, l'altération de la qualité de l'eau peut avoir un impact sur les activités marines et déterminer leur durée d'interruption, donc la perte économique liée. Par exemple, le mouillage ou la circulation des navires ne seront affectés qu'en cas de forte pollution avec présence de nappes de polluants susceptibles de souiller les coques. Dans ce cas, la perturbation pourra ne durer que quelques jours. À l'extrême opposé, certaines activités conchylicoles et certains établissements spécifiques (thalassothérapie, aquarium) pourront être durablement affectés par une pollution même diffuse (traces de polluants) qui altèrera la qualité de l'eau, indispensable à leur bon déroulement. La présence d'un seul établissement peut donc suffire à conférer une forte sensibilité à un site ou une portion de littoral. Cette méthode a été développée pour l'atlas du Finistère et appliquée à l'atlas du Morbihan en ne considérant que les filières professionnelles : industries, cultures marines, pêche, tourisme (CEDRE, 2005).

Tableau 4 : Méthodes d'évaluation de la sensibilité socio-économique aux pollutions marines/ Methods of assessment of socio-economic sensitivity to marine pollution

\begin{tabular}{|c|c|c|}
\hline $\begin{array}{l}\text { Type d'indice socio- } \\
\text { économique }\end{array}$ & Principe & $\begin{array}{l}\text { Exemple } \\
\text { d'application }\end{array}$ \\
\hline $\begin{array}{l}\text { Localisation des } \\
\text { activités }\end{array}$ & $\begin{array}{l}\text { Localisation, généralement ponctuelle, des activités } \\
\text { sensibles à une pollution marine par les } \\
\text { hydrocarbures } \\
=\text { pas de hiérarchisation }\end{array}$ & $\begin{array}{l}\text { Seine-Maritime } \\
1999\end{array}$ \\
\hline Somme des activités & $\begin{array}{l}\text { Somme des activités marines et littorales par } \\
\text { commune : les communes regroupant le plus grand } \\
\text { nombre d'activité seront les plus vulnérables }\end{array}$ & Manche en 2006 \\
\hline $\begin{array}{l}\text { Importance } \\
\text { activités }\end{array}$ & $\begin{array}{l}\text { Évaluation en fonction du nombre d'entreprises, du } \\
\text { nombre de salariés, de la production, da la capacité } \\
\text { d'accueil, du chiffre d'affaires, etc. }\end{array}$ & $\begin{array}{lll}\text { Côtes } & \text { d'Armor en } \\
2001 ; & \text { Manche en } \\
2006 & & \end{array}$ \\
\hline $\begin{array}{l}\text { Importance et } \\
\text { nombre d'activités }\end{array}$ & $\begin{array}{l}\text { Combine le nombre d'activités présentes et leur } \\
\text { importance socio-économique. }\end{array}$ & $\begin{array}{l}\text { IMO-IPIECA en } 2007 \\
\text { (Atlas } \quad \text { Afrique } \\
\text { GIWACAF) }\end{array}$ \\
\hline
\end{tabular}




\begin{tabular}{|l|l|l|}
\hline Durée d'interruption & $\begin{array}{l}\text { Évaluation de la durée d'interruption des activités } \\
\text { en fonction de l'intensité de la pollution (de } \\
\text { quelques heures à plusieurs années) }\end{array}$ & $\begin{array}{l}\text { Finistère et Morbihan } \\
\text { en } 2005\end{array}$ \\
\hline $\begin{array}{l}\text { Somme des activités } \\
\text { et durée } \\
\text { d'interruption }\end{array}$ & $\begin{array}{l}\text { Combine le nombre d'activités présentes et la durée } \\
\text { potentielle d'interruption (indice du CEDRE) }\end{array}$ & $\begin{array}{l}\text { Nord et Pas-deCalais } \\
\text { en 2004 }\end{array}$ \\
\hline Indice composite & $\begin{array}{l}\text { Pondération sur l'économie maritime, le tourisme, } \\
\text { les activités marchandes et non marchandes en } \\
\text { intégrant la saisonnalité des activités }\end{array}$ & $\begin{array}{l}\text { N. Levratto et I. } \\
\text { Clénceau (2005) } \\
\text { pour la Corse }\end{array}$ \\
\hline Indice global & $\begin{array}{l}\text { Construction d'un indice autour de quatre } \\
\text { composantes de la vulnérabilité socio-économique : } \\
\text { gestion, patrimoine, infrastructures et activités } \\
\text { socio-économiques }\end{array}$ & $\begin{array}{l}\text { P. Fattal (2008) pour } \\
\text { lîle de Noirmoutier }\end{array}$ \\
\hline
\end{tabular}

Certaines applications proposent un système de cotation visant à relier entre eux plusieurs de ces indices. Ainsi, dans la proposition formulée par l'omi et l'IPIECA (2007) pour la cartographie de la vulnérabilité côtière en Afrique, le nombre et l'importance des activités sont combinés, afin d'en hiérarchiser la vulnérabilité. Toute la difficulté de l'exercice consiste à apprécier à sa juste valeur l'importance relative des différentes activités, comme l'a aussi constaté E. Lagabrielle (2001).

\section{Sur les indices de sensibilité globale}

Plusieurs travaux menés pour la mise à jour d'atlas POLMAR ou dans un cadre plus expérimental se sont intéressés à la mise au point d'indices de sensibilité globale, intégrant des paramètres morphosédimentaires, écologiques et socio-économiques.

Une première tentative est à porter au crédit du CEDRE dans le cadre de l'élaboration de l'atlas de la Manche (Kerambrun et al., 1996). L'indice de vulnérabilité biomorphosédimentaire de L. Page-Jones considère la capacité de piégeage du sédiment, la rémanence ou durée de persistance du pétrole sur la côte, et la sensibilité écologique de la flore et de la faune du littoral considéré. Il intègre en quelque sorte l'ESI et la sensibilité écologique en proposant une pondération des trois paramètres exprimée par la formule suivante :

$\mathrm{V}=2 \mathrm{R}+\mathrm{K}+3 \mathrm{E}-5$

où $\mathrm{V}$ est l'indice de vulnérabilité, $\mathrm{K}$ la capacité de piégeage, $\mathrm{R}$ la rémanence et $\mathrm{E}$ la sensibilité écologique. En fonction de son importance, chaque paramètre se voit attribuer une valeur de 1 (faible) à 3 (forte) et une pondération. La constante - 5 est destinée contenir la valeur de l'indice entre 1 et 13.

Lors de l'élaboration de l'atlas des Côtes-d'Armor le CETMEF s'est attaché à ajouter la sensibilité socio-économique (Lagabrielle, 2001) à cet indice biomorphosédimentaire. Pour cela ont été rajoutés un coefficient $\mathrm{M}$ de mobilité de l'activité vis-à-vis de la pollution (les activités non mobiles étant considérées plus sensibles) et l'importance économique de l'activité $\mathrm{I}$, ces coefficients étant simplement cotés de 1 à 3 . Après pondération de chaque paramètre et division par une constante « 8 » destinée à ramener les valeurs de l'indice de 1 à 13 , la formule de cotation obtenue est la suivante : 

saisons. l'alimenter?

$\mathrm{Veco}=(3 \mathrm{M}+9 \mathrm{I}+6 \mathrm{~V}-10) / 8$

Constatant que l'indice de Lagabrielle ne prenait en compte que les activités qui exploitent les ressources marines (pêche, aquaculture, mytiliculture, etc.) un indice plus complet a été élaboré pour la réalisation de l'atlas de la Corse (Levratto et Clémenceau, 2005). Il intègre les autres activités marchandes liées directement ou non à la mer (clubs nautiques, compagnies de promenades en mer, etc.) ainsi que des activités bénéficiant de la proximité de la mer et pouvant être indirectement affectées par une pollution marine : hôtels, campings, mais aussi bars restaurants, autocaristes. La formulation de cet indice de vulnérabilité socio-économique s'écrit comme suit :

$\mathrm{V}=1 / 3\left(\mathrm{PC}+2 \mathrm{~A}_{\mathrm{D}}+2 \mathrm{~A}_{\mathrm{P}}+\mathrm{L}+\mathrm{P}-5\right)$

où $P C$ représentent les ports de commerce, $A_{d}$ les activités professionnelles liées directement à la mer, $A_{\mathrm{p}}$ les activités professionnelles qui bénéficient de la proximité de la mer, $L$ les activités de loisirs et $P$ la patrimonialité culturelle. La pondération est le fruit d'une série d'hypothèses et la constante $1 / 3$ permet de ramener la valeur de l'indice dans une échelle de 1 à 8 (en arrondissant les valeurs obtenues sur des valeurs entières). L'intérêt de cette démarche réside dans la possibilité d'exprimer la variabilité saisonnière de la vulnérabilité socio-économique, l'indice étant calculé pour deux

Enfin, dans le cadre de sa recherche, P. Fattal (2008) propose une intégration encore plus poussée intégrant une quarantaine de paramètres pour la production d'une dizaine d'indices thématiques couvrant les aspects morphosédimentaires, écologiques et socio-économiques qui lui permettent d'aboutir à un indice global qualifiant la sensibilité globale du littoral de Noirmoutier.

Il constate cependant que le calcul d'un indice synthétique de la vulnérabilité environnementale n'est pas chose aisée en raison de deux obstacles principaux liés à la disponibilité et à la qualité des données requises ainsi qu'à l'arbitraire des choix des cotations et des pondérations.

\section{Trouver un compromis entre exhaustivité et opérationnalité}

Entre le simple pointage des activités sur le littoral et la production d'un indice de sensibilité globale on voit que l'écart est grand. De fait, l'absence de standard traduit bien les questions posées par l'évaluation de la sensibilité socio-économique :

- Quelles activités considérer dans l'analyse (marchandes/non marchandes? directement/indirectement liées à la mer ? valeurs économique/valeur d'usage, etc.) ?

- Jusqu'où aller dans leur description (présence/absence, nombre d'emplois, chiffre d'affaires, variations saisonnières, etc.) ?

- Comment exprimer la valeur relative de ces activités pour permettre leur hiérarchisation (cotation et pondération)?

- Où trouver les données statistiques pertinentes et régulièrement mises à jour pour 
49 Le pointage présente l'avantage de la simplicité, mais ne permet pas réellement de hiérarchiser les enjeux. Par sa complexité intrinsèque, la production d'un indice global est quant à elle porteuse de plusieurs inconvénients :

50 L'augmentation du nombre de paramètres à prendre en compte dans le calcul nécessite d'accroître en proportion l'effort de collecte de données. Par conséquent, elle augmente aussi le risque de se heurter à l'indisponibilité de données précises, actualisées et accessibles pour l'ensemble des paramètres traités ou sur l'intégralité des territoires considérés.

51 L'adoption de système de cotation et de pondération sur la base d'hypothèses explicitées mais pas toujours évidentes à justifier est de nature à altérer la vocation d'objectivité d'un indice ;

52 Cette objectivité scientifique est-elle suffisante pour garantir l'utilité opérationnelle d'un indice ? La complexité de sa formulation peut rendre un indice peu explicite pour une partie de ses utilisateurs potentiels. Elle pose donc la question de son acceptation et de sa reconnaissance, comme base de décision dans un contexte opérationnel, qui doit nécessairement reposer sur une certaine forme de consensus.

53 Dans tous les cas, les indices visent à fournir une vision synthétique d'une réalité complexe. Mais ils en constituent également une interprétation. Jusqu'à quel point faut-il décrire cette complexité ? Si l'on reconnaît que les paramètres conjoncturels d'une marée noire varient d'un accident à l'autre et évoluent même en cours d'intervention et de traitement d'une pollution, la complexité devient difficile à appréhender et à anticiper.

54 Certes des outils de modélisation existent, mais ils s'attachent pour l'essentiel à simuler le comportement d'un polluant à la mer en fonction des facteurs hydrodynamiques (courants, houles), en vue de pouvoir suivre et prévoir l'évolution de la pollution pour planifier au mieux les opérations d'intervention (Bastien-Ventura et al., 2005). Ils peuvent également être mis en œuvre pour évaluer les dépenses liées aux marées noires (voir par exemple D.S. Etkin et al., 2002). Cependant, à ce niveau même l'incertitude reste grande, il s'agit bien de simulations, pas de prévision.

55 Dans les faits, le dispositif actuel de la lutte contre les pollutions marines fait largement appel à l'expertise. En plus de la vue d'ensemble qu'il offre, l'avis d'experts contribue sensiblement à affiner l'appréciation des enjeux en situation de crise et à pouvoir adapter les modalités d'intervention à ses spécificités. Le succès de l'intervention dépend donc en grande partie de la réactivité des équipes chargées de la lutte et repose généralement sur une approche très pragmatique de la situation.

56 Ainsi, s'ils ont vocation à fournir un éclairage et une hiérarchisation générale des paramètres pertinents pour la lutte antipollution, les indices ne se substituent pas à une expertise en situation de crise qui est celle préconisée par le Guide de révision des plans POLMAR du CEDRE en 2003.

57 De plus, un atlas de sensibilité à la pollution marine ne saurait être assimilé à un atlas de l'environnement littoral: il n'a pas vocation d'exhaustivité et doit rassembler uniquement les données utiles dans une perspective opérationnelle d'intervention contre les pollutions marines, tout en veillant à couvrir de manière homogène et exhaustive le littoral considéré. La consultation menée au sein de la MIMEL confirme que la simplicité de mise en œuvre et de compréhension d'un indice constitue un paramètre important, notamment pour en garantir la reproductibilité sur d'autres départements 
côtiers. La production d'un indice de sensibilité globale n'est donc pas recommandée. Basé sur l'agrégation de données très hétérogènes, par leur source comme par leur thème, il risque de devenir difficile à comprendre et donc à utiliser à des fins opérationnelles.

\section{Une proposition d'indice socio-économique pour les atlas normand}

\section{Principes méthodologiques}

Lors de la phase d'expérimentation menée en vue de la mise à jour de l'atlas de la Manche en 2006, nous avons proposé une série d'indices de sensibilité socioéconomique reposant sur un inventaire communal de neuf catégories d'activités liées à la mer et au littoral. Trois séries de paramètres ont été utilisées pour les décrire : somme des activités par commune; hiérarchisation en s'appuyant sur les volumes de production, sur la capacité d'accueil ou simplement sur le nombre d'entreprises ${ }^{12}$; indice d'interruption du CEDRE. Les indices testés utilisent ces paramètres indépendamment ou de manière combinée en respectant les recommandations formulées par G. Le Sage (2000) : toutes les données employées sont relativement aisées à obtenir et les méthodes de calculs restent simples (voir I. Le Berre et al., 2008 pour davantage de détails sur ces travaux).

Les expérimentations réalisées à partir de ces différentes méthodes et soumises à avis d'experts nous ont finalement conduit à adopter la version la plus simple (somme des activités par commune) car les résultats obtenus ne différaient pas de manière significative (Le Berre et al., 2008; Hariz, 2010). La durée d'interruption est cependant retenue dans deux cas: les prises d'eau, et les cultures marines. L'indice finalement adopté pour les atlas normands mis à jour en 2010 repose donc sur les principes suivants :

- Comptabilité du nombre d'activités économiques marchandes propres à l'espace marin et côtier présentes dans les communes du littoral ;

61 - Attribution de la note la plus élevée (10) à une commune, dès lors qu'une prise d'eau de mer y est implantée. Conformément au principe de l'indice d'interruption d'activités du CEDRE, nous avons différencié les prises d'eau conchylicoles des prises d'eau industrielles, de centres de thalassothérapie ou d'aquarium. Cela permet d'éviter les doubles comptes avec la rubrique «Cultures marines»;

62 - Attribution d'une note minimale de 5 aux communes dont l'estran fait l'objet d'une exploitation conchylicole. Cette cotation traduit un compromis entre la forte dépendance de cette activité vis-à-vis de la qualité d'eau de mer - et donc de sa forte sensibilité - et la possibilité de déplacement des cultures marines en dehors des sites pollués.

63 La production de notre indice est effectuée à partir de données simples à acquérir auprès d'organismes dûment répertoriés, seule l'absence ou la présence des activités et aménagements étant considérée (tableau 5). Naturellement, la cartographie de synthèse produite à partir de cet indice doit, dans un atlas de sensibilité, être proposée en plus des cartes thématiques pointant les activités (localisation précise, nature et 
importance). En effet les incidences opérationnelles sont différentes selon les types d'activités.

Tableau 5 : Paramètres employés pour établir l'indice de sensibilité socio-économique/Parameters used to determine the index of socio-economic sensitivity

\begin{tabular}{|c|c|c|c|c|}
\hline $\begin{array}{l}\text { Activités } \\
\text { economiques }\end{array}$ & Code & $\begin{array}{l}\text { Paramètres mesures } \\
\text { à l'échelle communale }\end{array}$ & Cotation & Source \\
\hline Prise d'eau & PRI & $\begin{array}{l}\text { présence d'une prise d'eau } \\
\text { industrielle, de thalassothérapie ou } \\
\text { d'aquarium }\end{array}$ & 10 & DDTM, DREAL \\
\hline Cultures marines & CMA & $\begin{array}{l}\text { présence d'un cadastre conchylicole } \\
\text { en face du trait de côte communal }\end{array}$ & 5 & DDTM \\
\hline $\begin{array}{l}\text { Pêches } \\
\text { professionnelles }\end{array}$ & PEC & $\begin{array}{l}\text { présence de débarquement de } \\
\text { pêches professionnelles }\end{array}$ & 1 & CRPMEM \\
\hline $\begin{array}{l}\text { Pêche à pied } \\
\text { professionnelle }\end{array}$ & PECp & $\begin{array}{l}\text { présence de pêche à pied } \\
\text { professionnelle }\end{array}$ & 1 & CRPMEM \\
\hline $\begin{array}{ll}\text { Transports } & \text { de } \\
\text { passagers } & \end{array}$ & PAS & $\begin{array}{l}\text { présence d'une activité de } \\
\text { transport de passagers }\end{array}$ & 1 & DREAL, CCI \\
\hline $\begin{array}{l}\text { Transports de } \\
\text { marchandises }\end{array}$ & MAR & $\begin{array}{l}\text { présence d'une activité de } \\
\text { transport de marchandises }\end{array}$ & 1 & DREAL, CCI \\
\hline Ports de plaisance & PLA & $\begin{array}{l}\text { présence de places de plaisance } \\
\text { (pontons, mouillages, échouages) }\end{array}$ & 1 & DDTM, CG \\
\hline $\begin{array}{l}\text { Hébergements } \\
\text { touristiques } \\
\text { marchands }\end{array}$ & HEB & $\begin{array}{l}\text { présence d'hébergements } \\
\text { touristiques marchands (résidences } \\
\text { secondaires exclues) }\end{array}$ & 1 & $\begin{array}{l}\text { Comité } \\
\text { départemental du } \\
\text { tourisme, CG }\end{array}$ \\
\hline $\begin{array}{l}\text { Loisirs nautiques } \\
\text { marchands }\end{array}$ & NAU & $\begin{array}{lcc}\text { présence } & \text { d'offres de } & \text { loisirs } \\
\text { nautiques } & \text { marchands } & \text { (clubs, } \\
\text { locations...) } & & \end{array}$ & 1 & $\begin{array}{l}\text { Comité } \\
\text { départemental du } \\
\text { tourisme, CG }\end{array}$ \\
\hline Lieu de baignade & BAI & $\begin{array}{l}\text { présence d'un site de mesure de la } \\
\text { qualité des eaux de baignades }\end{array}$ & 1 & DDASS \\
\hline
\end{tabular}


Figure 3 : Sensibilité socio-économique du littoral de la Manche/The socio-economic sensitivity of the Manche coastal zone

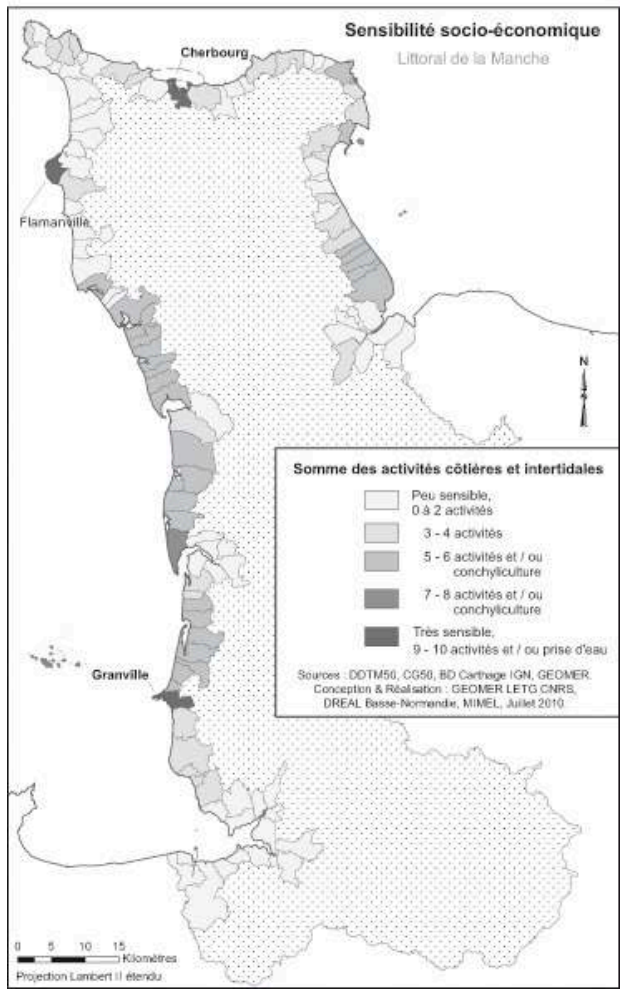

Figure 4 : Sensibilité socio-économique du littoral du Calvados/The socio-economic sensitivity of the Calvados coastal zone

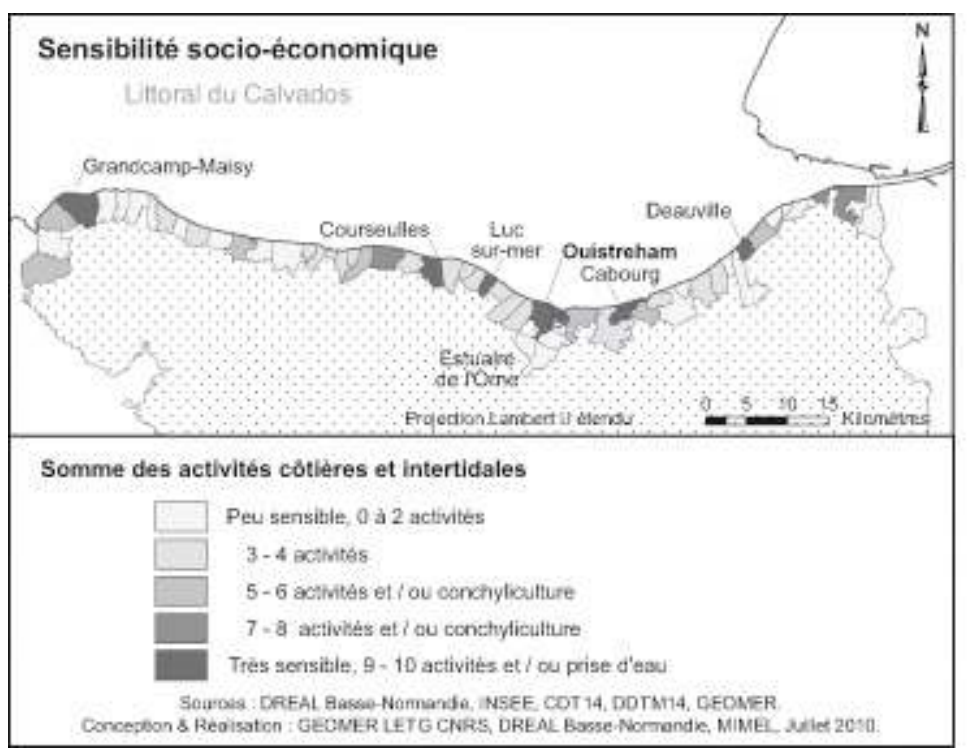

\section{Commentaire sur les cartes obtenues}

Dans la Manche (fig. 3) la méthode adoptée permet de mettre en évidence les communes dotées de prises d'eau liées à l'industrie (Flamanville), mais aussi à d'autres activités (aquarium de Cherbourg), celles dont l'économie est fortement orientée vers la mer et le littoral (Granville), ainsi que des communes à vocation touristique 
(Barneville-Carteret, Portbail, Agon-Coutainville, Saint-Vaast-la-Hougue, etc.) et/ou conchylicole (Anneville-sur-Mer, Coudeville-sur-Mer, etc.).

Dans le Calvados (fig. 4), cinq communes (Courseulles, Luc-sur-Mer, Ouistreham, Cabourg, Deauville) se démarquent en raison de la présence sur leur littoral d'une ou de plusieurs prises d'eau, pour la plupart liées à une activité de thalassothérapie. On note également la sensibilité forte de Grandcamp-Maisy qui accueille des activités côtières variées et en particulier la conchyliculture. Le résultat obtenu met encore en évidence les communes conchylicoles (Ver-sur-Mer, Meuvaine, Gefosse-Fontenay), ainsi que celle d'Honfleur grâce à sa vocation portuaire et touristique.

Les falaises du Pays de Caux, rendent peu accessible la majeure partie du littoral de Seine-Maritime (fig. 5), ce qui explique la concentration des activités aux embouchures des principaux cours d'eau. La carte produite illustre bien cette configuration en faisant ressortir les communes à vocation portuaire et industrielle (Le Havre naturellement, mais aussi Dieppe et Le Tréport) et conchylicole (Veules-les-Roses). Elle met surtout en évidence les communes abritant une prise d'eau, dont celles de Paluel et de Penly à la vocation maritime peu affirmée, mais qui accueillent chacune leur centrale nucléaire.

\section{Conclusion}

67 Les plans POLMAR doivent intégrer «un inventaire précis et hiérarchisé » des sites sensibles du littoral départemental sous la forme d'un atlas qui en constitue une annexe obligatoire. Etablis sous la responsabilité du Préfet, cet inventaire prend des formes variées selon les départements en fonction des moyens déployés, des données disponibles et des compétences et méthodes mises en œuvre. Pourtant sa vocation d'aide à la décision dans le contexte préparatoire et opérationnel de la lutte antipollution, justifie une certaine harmonisation de manière à bénéficier d'un niveau de connaissance et de modes de description et de hiérarchisation des enjeux littoraux standardisés d'un territoire à l'autre afin de pouvoir coordonner correctement les opérations d'intervention et de nettoyage.

L'analyse des atlas POLMAR disponibles sur le littoral français montre que l'estimation de la sensibilité géomorphologique repose le plus souvent sur l'adaptation locale de l'indice morphosédimentaire ESI, malgré ses limites pour les pollutions liées à des hydrocarbures lourds et visqueux. Par sa simplicité et sa logique, d'apparence évidente, il a été accepté très largement et de manière consensuelle par les maîtres d'œuvre des plans de prévention établis sur des littoraux du monde entier et dans des contextes très différents. L'estimation de la sensibilité écologique se base quant à elle sur plusieurs méthodes complémentaires en fonction des échelles adoptées et des données disponibles. La plus simple repose sur la superposition des zonages de protection environnementaux et sur leur addition pour définir la valeur patrimoniale accordée au littoral. Elle peut être complétée par une évaluation de la sensibilité des habitats et de l'avifaune, plus rarement des mammifères marins ce qui nécessiterait l'exploitation d'inventaires naturalistes qui ne sont pas systématiquement conduits sur les littoraux français. 
Figure 5 : Sensibilité socio-économique du littoral de la Seine-Maritime/The socio-economic sensitivity of the Seine-Maritime coastal zone

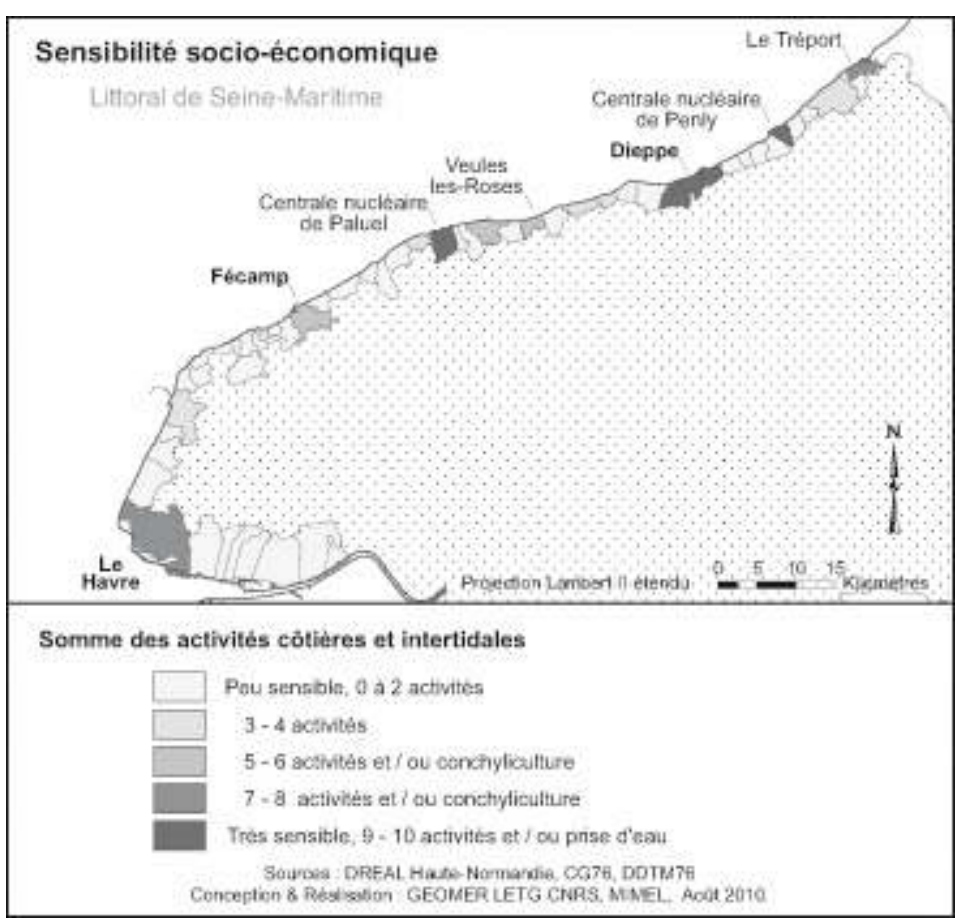

Le traitement du volet socio-économique reste quant à lui très variable, du simple inventaire des activités sensibles du littoral, jusqu'à la production d'indices composites élaborés sur une base économétrique. Si les premiers ne permettent pas de hiérarchiser les enjeux, comme spécifié dans les textes réglementaires encadrant le dispositif POLMAR, les derniers apparaissent relativement complexes à mettre en œuvre, ne seraitce que par le volume et la diversité des données à mobiliser. Reposant sur des systèmes élaborés de calcul et des formulations complexes de pondération, ils peuvent s'avérer difficiles à comprendre et à appliquer dans un contexte opérationnel posant ainsi la question de leur acceptabilité. C'est pourquoi, nous nous sommes attachés à rechercher un compromis satisfaisant entre simplicité de mise en œuvre et exhaustivité pour la mise à jour des atlas de sensibilité du littoral des trois départements normands.

Après une analyse des méthodes mises en œuvre dans les atlas POLMAR disponibles sur le littoral français, un indice socio-économique a été proposé et appliqué sur les trois atlas normands. Combinant la somme des activités présentes par communes et l'indice d'interruption des activités du CEDRE (2005), cette approche nous a permis de mettre en avant les principaux enjeux et la sensibilité socio-économique des communes littorales de Basse et Haute Normandie.

71 En tout état de cause, il convient d'insister sur le fait que malgré l'aide précieuse que les indices de sensibilité peuvent procurer aux décideurs et opérateurs de dépollution, la gestion des pollutions marines reste délicate. Elle se fait au cas par cas en fonction des orientations dictées par les indices et des circonstances de l'accident. Elle ne peut donc s'envisager sans l'avis d'experts. Ce principe est d'ailleurs parfaitement intégré dans le dispositif français de la lutte antipollution, puisque ces experts sont systématiquement consultés et font partie des organes de décision établis à différents niveaux lors de la mise en œuvre du plan POLMAR-TERRE. 
72 Naturellement, l'utilisation de ces indices de sensibilité et plus largement la mise à jour des atlas POLMAR repose sur la disponibilité de jeux de données actualisés et couvrant tout le littoral. Si ce n'est pas encore une réalité tout à fait concrète sur l'ensemble du littoral français, l'évolution est largement positive, grâce à la mise en place d'observatoires de l'environnement à différentes échelles (nationale, régionales) ${ }^{13}$, désormais encadrés par la Directive européenne INSPIRE qui «impose aux autorités publiques de rendre leurs données accessibles au public en les publiant sur Internet et de les partager entre elles ${ }^{14} »$. C'est bien le contexte dans lequel s'inscrivent nos travaux car la mise à jour des atlas POLMAR des départements de la Manche, du Calvados et de la Seine-Maritime constitue une application du pôle géomatique mer et littoral mis en œuvre au sein de la MIMEL. Son développement se poursuivra dorénavant au sein de la Direction interrégionale de la mer (DIRM), Manche-mer-du-Nord de la baie du Mont-Saint-Michel jusqu'à la frontière Belge.

Nous remercions vivement la DIREN (intégrée depuis le $1^{\text {er }}$ janvier 2010 à la DREAL), son directeur et son personnel, et la Préfecture de Basse-Normandie pour le soutien constant accordé à nos travaux, notamment par l'allocation d'une subvention de la MIMEL et du Fond national d'aménagement et de développement du territoire (FNADT).

Nos remerciements s'adressent également à l'ensemble des personnels des services déconcentrés de l'État en Haute et Basse-Normandie, ainsi qu'aux différents partenaires de la MIMEL qui, par leur contribution et l'intérêt portés à nos travaux ont grandement contribués à leur aboutissement.

Enfin, nous remercions très sincèrement nos deux relecteurs dont les commentaires bienveillants et constructifs ont contribué à améliorer sensiblement l'article proposé.

\section{BIBLIOGRAPHIE}

BASTIEN VENTURA C., GIRIN M., RAOUL-DUVAL J., 2005. Marées noires et environnement. Monaco, Institut Océanographique, $407 \mathrm{p}$.

BERNÉ S., DANTEC M.-H., D’OZOUVILLE J.-G., D'OZOUVILLE L., MARCHAND M., QUILFEN Y., TONDEREAU T., 1982. Veille écologique, vulnérabilité morphosédimentaire du littoral aux pollutions par hydrocarbures de la pointe Saint-Mathieu au sillon du Talbert, CNEXO, Centre Océanologique de Bretagne, Ministère de l'Environnement. $154 \mathrm{p}$.

BINARD R., TIMSIT O., GOURIÉ C., 2008. Vulnérabilité de l'avifaune côtière aux pollutions marines en Normandie, Rapport Groupe Ornithologique Normand/Conservatoire du littoral, $59 \mathrm{p}$.

BONNIEUX F., RAINELLI P., 2002, Évaluation des dommages des marées noires : une illustration à partir du cas de l'Erika et des pertes d'agrément des résidents, Economie et Statistique, n 357-358, p. 173-187.

CASTANEDO S., JUANES J.A., MEDINA R., PUENTE A., FERNANDEZ F., OLABARRIETA M, POMBO C., 2009. OIL SPILL VULNERABILITY ASSESSMENT INTEGRATING PHYSICAL, BIOLOGICAL AND SOCIO-ECONOMICAL ASPECTS : APPLICATION TO THE CANTABRIAN COAST (BAY OF BISCAY, SPAIN), JOURNAL OF ENVIRONMENTAL MANAGEMENT, VOL. 91, № 1, P. 149-159. 
CEDRE, 2003. GUIDE DE RÉVISION DES PLANS POLMAR-TERRE, BREST, CEDRE, [http://www.cedre.fr/fr/rejet/obligation/ guide_revision.pdf], $164 \mathrm{P}$.

CEDRE, 2005. ATLAS DU PLAN POLMAR DU DÉPARTEMENT DU FINISTÈRE, CD-ROM, [http://www.cedre.fr/fr/lutte/guide-elu/ cocopaq/atlas-sensibilite/index.htm].

CLARK R. B., 2002. Marine pollution, Oxford University Press, UK, 237 p.

DICKS B., ANSELL D. V., GUENETTE C. C., MOLLER T. H., SANTNER R. S., WHITE I. C., 2002. A review of problems posed by spills of heavy fuel oils. Actes du Third R \& D Forum on High-density Oil Spill Response, support CD, International Maritime Organization, Brest, France, 11-13 mars 2002. [http:// www.imo.org].

ETKIN, D. S., D. FRENCH-MCCAY, N. WHITTIER, S. SUBBAYYA, J. JENNINGS, 2002. Modeling of response, socioeconomic, and natural resource damage costs for hypothetical oil spill scenarios in San Francisco Bay. Proc. 25th Arctic \& Marine Oilspill Program Tech. Sem., p. 1075-1102. [http:// www.environmental-research.com/erc_papers/ERC_paper_9.pdf].

FATTAL P., 2008. POLLUTION DES CÔTES PAR LES HYDROCARBURES, RENNES, PUR, COLL. « ESPACE ET TERRITOIRES », 400 P.

FiCHAUT B., FATTAL P., 2000. Pourquoi le nettoyage de la marée noire de l'Erika est-il exceptionnellement long ?, Actes du colloque « Qui embruta el mar ? ", Barcelone, Espagne, 6-7 juin 2000, p. 121-128.

GUNDLACH E. R., HAYES M. O., 1978. VULNERABILITY OF COASTAL ENVIRONMENT TO OIL SPILL IMPACTS, MARINE TECHNICAL SOCIETY JOURNAL, VOL. $12, \mathrm{~N}^{\circ} 4$, P. $18-27$.

HARDEGEN M., GOURMELON F., BIORET F., MAGNANON S., 2001. La cartographie des habitats terrestres du réseau Natura 2000 en Bretagne. Mappemonde, vol. 64, n 1, p. 19-23. [http://www.mgm.fr/PUB/ Mappemonde/M401/Gourmelon.pdf].

HARIZ M. R., 2010. CONTRIBUTION À LA MISE À JOUR DU GUIDE DES DISPOSITIONS SPÉCIFIQUES POLMAR DE L'ORSEC DÉPARTEMENTAL: EXPÉRIMENTATION SUR LES ATLAS DE HAUTE ET DE BASSE NORMANDIE, MÉMOIRE DE MASTER 2 EGEL, MENTION RECHERCHE, UNIVERSITÉ DE BRETAGNE OCCIDENTALE (IUEM-UBO), 88 P.

KERAMBRUN L., DAVID L., PAGE-JONES L., REBOUT C., 1996. ATLAS POLMAR-TERRE DU DÉPARTEMENT DE LA MANCHE, BREST, CEDRE DPNM, 70 P.

LACAZE J.-C., 1980. La pollution pétrolière en milieu marin, Paris, Masson, 132 p.

LAGABRIELLE E., 2001. Plan POLMAR-TERRE des Côtes-d'Armor, mise en place d'un SIG, Mémoire de MST aménagement du territoire, IGARUN (Université de Nantes) - CETMEF, Nantes, 187 p.

LE BERRE I., GOURIOU V., NÉDELEC M., ALBRECHT M., 2005, SIG et lutte antipollution marine par les hydrocarbures, dans Gourmelon F., RoBin M. (dir.), SIG et littoral, éd. Hermès-Lavoisier, coll. «IGAT », p. 237-256.

LE BERRE I., QUEMMERAIS F., FICHAUT B., 2008. RÉVISION DE L'ATLAS POLMAR-TERRE DU DÉPARTEMENT DE LA MANCHE: VERS UN SIG OPÉRATIONNEL INTERSERVICES, CYBERGEO, $\mathrm{N}^{\circ}$ 422, [http://www.cybergeo.eu/index18082.html].

LE BERRE I., LE TIXERANT M., NOGUES L., 2010. Information géographique et gestion intégrée des zones côtières : analyse et expérimentation d'un SIG inter-services mer et littoral. Rapport d'expertise scientifique, MIMEL/DREAL de Basse-Normandie/GEOMER UMR 6554 CNRS LETG (IUEM-UBO), 100 p. [http://hal.archives-ouvertes.fr/index.php? action_todo=search\&view_this_doc=hal-00535795\&version=1\&halsid=8efjmitnq7splsbiu8v1odibq1]. 
LE SAGE G., 2000, Typologie des communes littorales selon leur portefeuille d'activités « littorales ». Groupement de la statistique publique en Languedoc Roussillon - dossier littoral, INSEE, [http:// www.languedoc-roussillon.ecologie.gouv.fr/publication/litto/inseetypologie.htm].

LEVRATTO N., CLÉMENCEAU I., 2005, Élaboration d'un indice de sensibilité socio-économique d'un littoral : une application au cas de la région Corse, Vertigo, vol. 6, $\mathrm{n}^{\circ}$ 3, [http:// www.vertigo.uqam.ca/].

LITTLE D. I., DEAKIN T., FICHAUT B., MEECH R. J., 2003. Some observations on heavy fuel oil spills : trends, impacts and comparisons with crude oil spills. Actes du colloque $d u 26^{\mathrm{e}}$ Arctic and Marine Oil Spill Program Technical seminar, Victoria, Colombie Britannique, Canada, Juin 2003, p. 971-999.

MICHEL J., DAHLIN J., 1997. Guidelines for developing digital Environmental Sensibility Index atlases and databases. Seattle, NOAA, 43 P.

NOAA, 2002. ENVIRONMENTAL SENSITIVITY INDEX GUIDELINES, VERSION 3.0, HAZARDOUS MATERIALS RESPONSE AND ASSESSMENT DIVISION, NOAA, 89 P.

OMI/IPIECA, 2007. GUIDE POUR LA CARTOGRAPHIE DE LA VULNÉRABILITÉ DES CÔTES AUX POLLUTIONS MARITIMES ACCIDENTELLES ET SON INTÉGRATION DANS LES SYSTÈMES DE PRÉPARATION À LA LUTTE ET DE LUTTE DANS LA RÉGION DE L'AFRIQUE DE L'OUEST ET DU CENTRE (GIWACAF), The Global Initiative for West and Central Africa (GI WACAF), [http://giwacaf.org/].

BASTIEN VENTURA C., GIRIN M., RAOUL-DUVAL J., 2005. Marées noires et environnement. Monaco, Institut Océanographique, $407 \mathrm{p}$.

BERNÉ S., DANTEC M.-H., D'OZOUVILLE J.-G., D’OZOUVILLE L., MARCHAND M., QUILFEN Y., TONDEREAU T., 1982. Veille écologique, vulnérabilité morphosédimentaire du littoral aux pollutions par hydrocarbures de la pointe Saint-Mathieu au sillon du Talbert, CNEXO, Centre Océanologique de Bretagne, Ministère de l'Environnement. 154 p.

BINARD R., TIMSIT O., GOURIÉ C., 2008. Vulnérabilité de l'avifaune côtière aux pollutions marines en Normandie, Rapport Groupe Ornithologique Normand/Conservatoire du littoral, 59 p.

BONNIEUX F., RAINELLI P., 2002, Évaluation des dommages des marées noires : une illustration à partir du cas de l'Erika et des pertes d'agrément des résidents, Economie et Statistique, n 357-358, p. 173-187.

CASTANEDO S., JUANES J.A., MEDINA R., PUENTE A., FERNANDEZ F., OLABARRIETA M, POMBO C., 2009. OIL SPILL VULNERABILITY ASSESSMENT INTEGRATING PHYSICAL, BIOLOGICAL AND SOCIO-ECONOMICAL ASPECTS : APPLICATION TO THE CANTABRIAN COAST (BAY OF BISCAY, SPAIN), JOURNAL OF ENVIRONMENTAL MANAGEMENT, VOL. 91, N 1 , P. 149-159.

CEDRE, 2003. GUIDE DE RÉVISION DES PLANS POLMAR-TERRE, BREST, CEDRE, [http://www.cedre.fr/fr/rejet/obligation/ quide_revision.pdf], $164 \mathrm{P}$.

CEDRE, 2005. ATLAS DU PLAN POLMAR DU DÉPARTEMENT DU FINISTÈRE, CD-ROM, [http://www.cedre.fr/fr/lutte/guide-elu/ cocopaq/atlas-sensibilite/index.htm].

CLARK R. B., 2002. Marine pollution, Oxford University Press, UK, 237 p.

DICKS B., ANSELL D. V., GUENETTE C. C., MOLLER T. H., SANTNER R. S., WHITE I. C., 2002. A review of problems posed by spills of heavy fuel oils. Actes du Third R \& D Forum on High-density Oil Spill Response, support CD, International Maritime Organization, Brest, France, 11-13 mars 2002. [http:// www.imo.org].

ETKIN, D. S., D. FRENCH-MCCAY, N. WHITTIER, S. SUBBAYYA, J. JENNINGS, 2002. Modeling of response, socioeconomic, and natural resource damage costs for hypothetical oil spill scenarios in San 
Francisco Bay. Proc. 25th Arctic \& Marine Oilspill Program Tech. Sem., p. 1075-1102. [http:// www.environmental-research.com/erc_papers/ERC_paper_9.pdf].

FATTAL P., 2008. POLLUTION DES CÔTES PAR LES HYDROCARBURES, RENNES, PUR, COLL. « ESPACE ET TERRITOIRES », 400 P.

FICHAUT B., FATTAL P., 2000. Pourquoi le nettoyage de la marée noire de l'Erika est-il exceptionnellement long ?, Actes du colloque « Qui embruta el mar ? ", Barcelone, Espagne, 6-7 juin 2000, p. 121-128.

GUNDLACH E. R., HAYES M. O., 1978. VULNERABILITY OF COASTAL ENVIRONMENT TO OIL SPILL IMPACTS, MARINE TECHNICAL SOCIETY JOURNAL, VOL. $12, \mathrm{~N}^{\circ} 4$, P. $18-27$.

HARDEGEN M., GOURMELON F., BIORET F., MAGNANON S., 2001. La cartographie des habitats terrestres du réseau Natura 2000 en Bretagne. Mappemonde, vol. 64, n 1, p. 19-23. [http://www.mgm.fr/PUB/ Mappemonde/M401/Gourmelon.pdf].

HARIZ M. R., 2010. CONTRIBUTION À LA MISE À JOUR DU GUIDE DES DISPOSITIONS SPÉCIFIQUES POLMAR DE L'ORSEC DÉPARTEMENTAL: EXPÉRIMENTATION SUR LES ATLAS DE HAUTE ET DE BASSE NORMANDIE, MÉMOIRE DE MASTER 2 EGEL, MENTION RECHERCHE, UNIVERSITÉ DE BRETAGNE OCCIDENTALE (IUEM-UBO), $88 \mathrm{P}$.

KERAMBRUN L., DAVID L., PAGE-JONES L., REBOUT C., 1996. ATLAS POLMAR-TERRE DU DÉPARTEMENT DE LA MANCHE, BREST, CEDRE DPNM, 70 P.

LACAZE J.-C., 1980. La pollution pétrolière en milieu marin, Paris, Masson, 132 p.

LAGABRIELlE E., 2001. Plan POLMAR-TERRE des Côtes-d'Armor, mise en place d'un SIG, Mémoire de MST aménagement du territoire, IGARUN (Université de Nantes) - CETMEF, Nantes, 187 p.

LE BERRE I., GOURIOU V., NÉDELEC M., ALBRECHT M., 2005, SIG et lutte antipollution marine par les hydrocarbures, dans GouRMELON F., RoBin M. (dir.), SIG et littoral, éd. Hermès-Lavoisier, coll. «IGAT », p. 237-256.

LE BERRE I., QUEMMERAIS F., FICHAUT B., 2008. RÉVISION DE L'ATLAS POLMAR-TERRE DU DÉPARTEMENT DE LA MANCHE: VERS UN SIG OPÉRATIONNEL INTERSERVICES, CYBERGEO, Nº 422, [http://www.cybergeo.eu/index18082.html].

LE BERRE I., LE TIXERANT M., NOGUES L., 2010. Information géographique et gestion intégrée des zones côtières : analyse et expérimentation d'un SIG inter-services mer et littoral. Rapport d'expertise scientifique, MIMEL/DREAL de Basse-Normandie/GEOMER UMR 6554 CNRS LETG (IUEM-UBO), 100 p. [http://hal.archives-ouvertes.fr/index.php? action_todo=search\&view_this_doc=hal-00535795\&version=1\&halsid=8efjmitnq7splsbiu8v1odibq1]. LE SAGE G., 2000, Typologie des communes littorales selon leur portefeuille d'activités « littorales ». Groupement de la statistique publique en Languedoc Roussillon - dossier littoral, INSEE, [http:// www.languedoc-roussillon.ecologie.gouv.fr/publication/litto/inseetypologie.htm]. LEVRATTO N., CLÉMENCEAU I., 2005, Élaboration d'un indice de sensibilité socio-économique d'un littoral : une application au cas de la région Corse, Vertigo, vol. 6, n 3, [http:// www.vertigo.uqam.ca/].

LITTLE D. I., DEAKIN T., FICHAUT B., MEECH R. J., 2003. Some observations on heavy fuel oil spills : trends, impacts and comparisons with crude oil spills. Actes du colloque du $26^{\mathrm{e}}$ Arctic and Marine Oil Spill Program Technical seminar, Victoria, Colombie Britannique, Canada, Juin 2003, p. 971-999.

MICHEL J., DAHLIN J., 1997. Guidelines for developing digital Environmental Sensibility Index atlases and databases. Seattle, NOAA, $43 \mathrm{P}$.

NOAA, 2002. ENVIRONMENTAL SENSITIVITY INDEX GUIDELINES, VERSION 3.0, HAZARDOUS MATERIALS RESPONSE AND ASSESSMENT DIVISION, NOAA, 89 P. 
OMI/IPIECA, 2007. GUIDE POUR LA CARTOGRAPHIE DE LA VULNÉRABILITÉ DES CÔTES AUX POLLUTIONS MARITIMES ACCIDENTELLES ET SON INTÉGRATION DANS LES SYSTÈMES DE PRÉPARATION À LA LUTTE ET DE LUTTE DANS LA RÉGION DE L'AFRIQUE DE L'OUEST ET DU CENTRE (GIWACAF), The Global Initiative for West and Central Africa (GI WACAF), [http://giwacaf.org/].

\section{ANNEXES}

CG Conseil général

CBNB Conservatoire botanique national de Brest

CEL Conservatoire du littoral et des rivages lacustres

CRPMEM Comité régional des pêches maritimes et de l'élevage en mer

DDAM Direction départementale des affaires maritimes

DDE Direction départementale de l'équipement

DDTM Direction départementale des territoires et de la mer (remplace les DDE)

DIREN Direction régionale de l'environnement

DRAM Direction régionale des affaires maritimes

DRE Direction régionale de l'Equipement

\section{DREAL}

Direction régionale de l'environnement, de l'aménagement et du logement (remplace les DRIRE, DRE et DIREN)

DRIRE Direction régionale de l'industrie, de la recherche et de l'environnement

INSEE Institut national de la statistique et des études économiques

IFREMER Institut français de recherche pour l'exploitation de la mer

SHOM Service hydrographique et océanographique de la marine

SRC Section régionale conchylicole

\section{NOTES}

1. Circulaire du 17 décembre 1997 relative à la lutte contre les pollutions accidentelles du milieu marin et aux plans de secours spécialisés POLMAR; Instruction du 2 avril 2001 relative à l'intervention des pouvoirs publics en cas d'accidents maritimes majeurs ; Instruction du 4 mars 2002 relative à la lutte contre la pollution du milieu marin (documentation nationale POLMAR).

2. La loi $n^{\circ}$ 2004-811 du 13 août 2004 de modernisation de la sécurité civile et les décrets d'application $n^{\circ}$ 2005-1156 Plan Communal de Sauvegarde (P.C.S.), n 2005-1157 plan ORSEC et $n^{\circ}$ 2005-1158 Plan Particulier d'Intervention (PPI) du 13 septembre 2005, puis le Décret $n^{\circ}$ 2005-1157 du 13 septembre 2005 relatif au plan ORSEC, et enfin la Circulaire INT/E/06/00120/C du ministère de l'Intérieur du 29 décembre 2006 et le Guide ORSEC départemental transforment en profondeur l'organisation de préparation et de lutte contre les pollutions majeures du littoral. En premier lieu, tous les Plans de Secours Spécialisés disparaissent, pour devenir des volets spécifiques du plan ORSEC départemental. 
3. La mission de la MIMEL s'est achevée avec ce projet... Les travaux entrepris dans ce cadre devraient être poursuivis par la Direction régionale de la mer (DIRMER) et étendus à l'ensemble de la façade Manche-Mer du Nord.

4. Cf. note 1 .

5. Cf. par exemple [http://www.pollutions-marines-normandie.fr/carto/].

6. [http://giwacaf.org/sensitivity.asp].

7. Cf. [http://www.cedre.fr/fr/lutte/guide-elu/cocopaq/atlas-sensibilite/index.htm].

8. Cf. [http://www.nord-pas-de-calais.ecologie.gouv.fr/rubrique.php3?id_rubrique=166].

9. Voir site du Conservatoire botanique national de Brest, [http://www.cbnbrest.fr/ botalittoral/].

10. Voir par exemple le site de la NOAA [http://response.restoration.noaa.gov/], ou celui de l'Australian Marine Safety Authority [http://www.amsa.gov.au/ Marine_Environment_Protection/National_plan/]

11. Lien vers le programme ATRACTIV : [http://www.conservatoire-du-littoral.fr/front/ process/Content.asp?rub=9\&rubec $=66]$.

12. Valeurs plus aisées à obtenir que le chiffre d'affaires ou les emplois générés par ces activités qui possèdent une valeur stratégique et restent souvent confidentielles.

13. Nous pensons notamment à l'observatoire du littoral [http://www.littoral.ifen.fr/], au Crig PACA [http://www.crige-paca.org/], au GéoBretagne [http://www.geobretagne.fr/], et naturellement à la composante littorale du Géoportail national [http:// www.geolittoral.equipement.gouv.fr/].

14. Extrait de la Directive INSPIRE 2007/2/CE du 14 mars 2007 [inspire.jrc.ec.europa.eu/].

\section{RÉSUMÉS}

Établis dans le cadre du volet Polmar-terre des plans Orsec, les atlas de sensibilité des littoraux doivent fournir " un inventaire précis et hiérarchisé des zones à protéger en priorité ». Les atlas existants sont produits à l'échelle départementale et, de fait, présentent une grande hétérogénéité de formats, de contenus ainsi que de méthodes de représentation et de hiérarchisation des enjeux. À partir de l'expérience acquise lors de la mise à jour des trois atlas normands, cet article propose des éléments de réflexion pour harmoniser les atlas de sensibilité et les indices employés, notamment pour faciliter la préparation des interventions concernant plusieurs départements.

Prepared in the framework of the French Polmar-terre oil spill contingency plan, sensitivity atlas must provide a detailed inventory of areas to protect in priority. As their production is under the supervision of local authorities (département), the available atlases are quite heterogeneous in their formats, their contents and their methods of classification and representation of sites and stakes. This paper is based on the experience acquired while updating three sensitivity atlases of Normandy coastal zone. It aims to provide practical considerations about the standardization of 
sensitivity atlases and the indexes that are used to assess coastal zone stakes. We hope to contribute to a better integration of contingency plans at interregional scale.

\section{INDEX}

Index géographique : Normandie

Keywords : oil spill, pollution, sensitivity, atlas

Mots-clés : pollution, sensibilité, marée noire, atlas

\section{AUTEURS}

\section{IWAN LE BERRE}

Géomer - UMR 6554 LETG CNRS (Université de Bretagne Occidentale), Institut Universitaire Européen de la Mer, Technopôle Brest-Iroise, place Nicolas-Copernic - 29285 Plouzané, France iwan.leberre@univ-brest.fr

\section{MOHAMED RASSIM HARIZ}

Géomer - UMR 6554 LETG CNRS (Université de Bretagne Occidentale), Institut Universitaire Européen de la Mer, Technopôle Brest-Iroise, place Nicolas-Copernic - 29285 Plouzané, France h.med.racim@hotmail.com

\section{LAURENCE DAVID}

Géomer - UMR 6554 LETG CNRS (Université de Bretagne Occidentale), Institut Universitaire Européen de la Mer, Technopôle Brest-Iroise, place Nicolas-Copernic - 29285 Plouzané, France laurence.david@univ-brest.fr

\section{LOÏC NOGUES}

Géomer - UMR 6554 LETG CNRS (Université de Bretagne Occidentale), Institut Universitaire Européen de la Mer, Technopôle Brest-Iroise, place Nicolas-Copernic - 29285 Plouzané, France loicnogues@hotmail.fr 